\title{
The Scattering of Certain Yang-Mills Fields *
}

\author{
Robert T. Glassey ${ }^{1 \star \star}$ and Walter A. Strauss ${ }^{2}$ \\ 1 Department of Mathematics, Indiana University, Bloomington, IN 47405, USA \\ 2 Department of Mathematics, Brown University, Providence, RI 02912, USA
}

\begin{abstract}
The Yang-Mills fields considered by us in an earlier paper are asymptotically non-interacting. Also any free field is an incoming field for some Yang-Mills field.
\end{abstract}

\section{Introduction}

Interest in the classical solutions of the Yang-Mills equations in Minkowski space has grown in recent years. The definitive global existence theorem (solution of the Cauchy problem) has been found by Eardley and Moncrief [3], following the local theorem of Segal [8]. Other existence results and methods appear in [6], [4] and [1]. However, the scattering problem has so far been left untouched. Christodoulou's transform method can be used to derive some decay properties. In [5] we showed how the conformal invariance directly implies certain asymptotic properties of the fields, in particular, local decay of the energy.

In Sect. 1 of this paper we use these asymptotic properties, and the special properties of the class of solutions discussed in [6], to prove that these solutions $\alpha$ are asymptotically free fields in the energy norm. In Sect. 3 we show that any free field $\alpha_{-}$of our special type is the incoming field of some $\alpha$. In Sect. 2 we derive some explicit pointwise bounds needed in the proof.

Our class of solutions is defined by a condition of the Polyakov-t'Hooft type for the gauge group $\mathrm{SU}(2)$. We emphasize that there is no restriction on the size of the solutions we consider. Specifically, the gauge potentials have the form

$$
A^{k}=\alpha(r, t) v^{k} \quad(k=1,2,3), \quad A^{0}=0 .
$$

where $t$ is time, $x \in \mathbb{R}^{3}, r=|x|$ and $v^{k}$ are certain vectors (see [6]). Such a field belongs to both the temporal and Coulomb gauges. The field equations reduce to a single scalar wave equation (see (1) below). Although this equation appears rather innocuous, its asymptotic analysis is surprisingly non-trivial due to the singularity at the origin. The free fields we consider are simply solutions of the Yang-Mills

* Research supported in part by NSF MCS81-21487 and NSF MCS82-01340

$\star \star \quad$ Alfred P. Sloan Research Fellow 
equations with vanishing coupling constant; that is, they satisfy the same equation but linearized around zero.

Thus physically interesting phenomena such as solitons appear to be absent from the pure Yang-Mills equations. It seems that a Higgs mechanism is necessary to make them appear.

\section{Asymptotic Freedom of the Yang-Mills Fields}

We consider the solutions of the real scalar equation

$$
\alpha_{t t}-\alpha_{r r}-\frac{2}{r} \alpha_{r}+\frac{2}{r^{2}} \alpha-\frac{3}{r} \alpha^{2}+\alpha^{3}=0
$$

Denote the energy density by

$$
e=e(r, t)=\frac{1}{2} \alpha_{t}^{2}+\frac{1}{2} \alpha_{r}^{2}+\frac{1}{4} \alpha^{2}\left(\frac{2}{r}-\alpha\right)^{2}
$$

and the inversional density by

$$
I=I(r, t)=\frac{1}{2}\left(t^{2}+r^{2}\right)\left(\alpha_{t}^{2}+\alpha_{r}^{2}\right)+2 t r \alpha_{t} \alpha_{r}+2 t \alpha_{t} \alpha-\alpha^{2}+\frac{1}{4}\left(t^{2}+r^{2}\right) \alpha^{2}\left(\frac{2}{r}-\alpha\right)^{2} .
$$

Furthermore we denote $x \in \mathbb{R}^{3}, r=|x|$ and $d x=4 \pi r^{2} d r$. Let

$$
\|\alpha\|_{*}^{2}=\int\left(\alpha_{t}^{2}+\alpha_{r}^{2}+\frac{2}{r^{2}} \alpha^{2}\right) d x
$$

at any time $t$, where the integration is over all of space $\mathbb{R}^{3}$. Let $\mathscr{H}$ be the Hilbert space of Cauchy data provided with this norm. That is, $\mathscr{H}=\widehat{H}_{r}^{1} \oplus L^{2}$ in the notation of [6].

Theorem 1. (a) If $\int e d x<\infty$ when $t=0$, then there is a unique solution $\alpha=\alpha(r, t)$ of $(1)$ with given Cauchy data at $t=0$ such that $\alpha \in C(\mathbb{R} ; \mathscr{H})$, the energy $\int e d x$ is independent of time and

(i) $B^{2}=\iint \alpha^{2}\left(\frac{2}{r}-\alpha\right)^{2} \frac{1}{r} d x d t<\infty$

(integral taken over all space-time).

(b) In case $\int\left(1+r^{2}\right) e d x<\infty$ when $t=0$, we have

(ii) $\int I(r, t) d x \leqq \int I(r, 0) d x<\infty$.

Furthermore, $\int\left(1+r^{2}\right) e d x<\infty$ for all time and

(iii) $\int_{r<\theta t} e d x+\int \alpha^{2}\left(\frac{2}{r}-\alpha\right)^{2} d x=0\left(\frac{1}{t^{2}}\right)$

for large $t$, for any $\theta<1$.

Proof. See [6]. For smooth $\alpha$ the last statements follow from (ii) because I can be rewritten as

$$
4 I=(t+r)^{2}\left(\alpha_{t}+\alpha_{r}+\frac{\alpha}{r}\right)^{2}+(t-r)^{2}\left(\alpha_{t}-\alpha_{r}-\frac{\alpha}{r}\right)^{2}
$$




$$
+\left(t^{2}+r^{2}\right) \alpha^{2}\left(\frac{2}{r}-\alpha\right)^{2}-2 r^{-2}\left[\left(t^{2}+r^{2}\right) r \alpha^{2}\right]_{r},
$$

where the last term drops out upon integration. Hence

$$
\int_{r<\theta t}\left[\alpha_{t}^{2}+\left(\alpha_{r}+\frac{\alpha}{r}\right)^{2}\right] d x=0\left(t^{-2}\right) .
$$

But for $R \leqq \theta t$

$$
\int_{r<R}\left(\alpha_{r}+\frac{\alpha}{r}\right)^{2} d x=\int_{r<R}\left[\alpha_{r}^{2}+\frac{1}{r^{2}}\left(r \alpha^{2}\right)_{r}\right] d x,
$$

whence

$$
\int_{r<R} \alpha_{r}^{2} d x+4 \pi R \alpha^{2}(R, t)=0\left(t^{-2}\right) .
$$

By a free solution we mean a solution of the equation

$$
L \beta \equiv \beta_{t t}-\beta_{r r}-\frac{2}{r} \beta_{r}+\frac{2}{r^{2}} \beta=0
$$

which is equation (1) with the nonlinear terms dropped. The main result of this section is

Theorem 2. Given a solution $\alpha$ of $(1)$ with $\int\left(1+r^{2}\right) e d x<\infty$. Then there exist a unique pair of free solutions $\alpha_{-}$and $\alpha_{+}$such that

$$
\left\|\alpha-\alpha_{ \pm}\right\|_{*} \rightarrow 0 \text { as } t \rightarrow \pm \infty \text {. }
$$

Lemma 1. As $t \rightarrow \infty$ we have

(a) $\sup _{r \leqq \theta t} r \alpha^{2}=0\left(t^{-2}\right)$ for $\theta<1$,

(b) $\int \alpha^{2} d x=0(\ln t)$,

(c) $\int \frac{\alpha^{2}}{r^{2}} d x=0\left(t^{-2} \ln t\right)$,

(d) $\sup _{0 \leqq r \leqq \infty} r \alpha^{2}=0\left(t^{-1}(\ln t)^{1 / 2}\right)$.

Proof. (a) is immediate from (3). Next we rewrite $I$ again as

$$
\begin{aligned}
& I=\frac{1}{2}\left(t \alpha_{t}+r \alpha_{r}+\alpha\right)^{2}+\frac{1}{2}\left(r \alpha_{t}+t \alpha_{r}\right)^{2}+\frac{1}{4}\left(t^{2}+r^{2}\right) \alpha^{2}\left(\frac{2}{r}-\alpha\right)^{2} \\
& \quad+t \alpha_{t} \alpha-\frac{1}{2 r^{2}}\left[r^{3} \alpha^{2}\right]_{r} .
\end{aligned}
$$

In this expression the first three terms are non-negative and the last term integrates to zero. So by Theorem 1(b) $t \int \alpha_{t} \alpha d x<c$. This implies (b). By (b), $\int_{r>\theta t} \frac{\alpha^{2}}{r^{2}} d x=$ 
$0\left(t^{-2} \ln t\right)$. Next we write

$$
\alpha^{2}=\left(r \alpha^{2}\right)_{r}-2 r \alpha_{r} \alpha
$$

whence

$$
\begin{aligned}
\int_{0}^{R} \frac{\alpha^{2}}{r^{2}} d x & =\left.4 \pi r \alpha^{2}\right|_{0} ^{R}-2 \int_{r<R} \alpha_{r} \frac{\alpha}{r} d x \\
& \leqq 4 \pi R \alpha^{2}(R, t)+2 \int_{r<R} \alpha_{r}^{2} d x+\frac{1}{2} \int_{r \leq R} \frac{\alpha^{2}}{r^{2}} d x .
\end{aligned}
$$

The last term is moved to the left side. The other terms are $0\left(t^{-2}\right)$ by (a) and (3), if $R \leqq \theta t$. This proves (c). Integrating $\left(^{*}\right)$ once again, we have

$$
\begin{aligned}
r \alpha^{2} & =\int \frac{\alpha^{2}}{r^{2}} d x+2 \int \alpha_{r} \frac{\alpha}{r} d x \\
& \leqq \int \frac{\alpha^{2}}{r^{2}} d x+2\left(\int \alpha_{r}^{2} d x\right)^{1 / 2}\left(\int \frac{\alpha^{2}}{r^{2}} d x\right)^{1 / 2} \\
& =0\left(t^{-2} \ln t+\left[t^{-2} \ln t\right]^{1 / 2}\right)
\end{aligned}
$$

which proves (d).

\section{Lemma 2.}

$$
\int\left\{\left[\frac{3}{r} \alpha^{2}-\alpha^{3}\right]^{2} d x\right\}^{1 / 2} d t<\infty
$$

Proof. We write

$$
\frac{3}{r} \alpha^{2}-\alpha^{3}=\frac{3}{2} \alpha^{2}\left(\frac{2}{r}-\alpha\right)+\frac{1}{2} \alpha^{3},
$$

and estimate the $L^{2}$ norm of each term on the right separately. First,

$$
\begin{aligned}
\int \alpha^{6} d x & \leqq \sup r^{2} \alpha^{4} \cdot \int \frac{\alpha^{2}}{r^{2}} d x \\
& =0\left(t^{-4} \ln ^{2} t\right),
\end{aligned}
$$

by Lemma 1 (c) and (d). Second,

$$
\begin{aligned}
A^{2}(t) & \equiv \int_{r<t / 2} \alpha^{4}\left(\frac{2}{r}-\alpha\right)^{2} d x \\
& \leqq \int \alpha^{2}\left(\frac{2}{r}-\alpha\right)^{2} \frac{1}{r} d x \cdot \sup _{r<t / 2} r \alpha^{2},
\end{aligned}
$$

so that by Lemma 1(a)

$$
\int_{1}^{\infty} A(t) d t \leqq c \int_{1}^{\infty}\left[\int \alpha^{2}\left(\frac{2}{r}-\alpha\right)^{2} \frac{1}{r} d x\right]^{1 / 2} \frac{d t}{t} \leqq c B<\infty .
$$


Finally,

$$
\begin{aligned}
\int_{r>t / 2} \alpha^{4}\left(\frac{2}{r}-\alpha\right)^{2} d x & \leqq \int \alpha^{2}\left(\frac{2}{r}-\alpha\right)^{2} d x \cdot \sup _{r>t / 2} \frac{1}{r} \cdot \sup r \alpha^{2} \\
& =0\left(t^{-4} \ln ^{1 / 2} t\right)
\end{aligned}
$$

by Lemma 1(d) and Theorem 1(b).

Proof of Theorem 2. Let $R(t)$ denote the Riemann operator for the free equation (4). This means that the solution $\beta$ of (4) with the initial data $\beta(r, 0) \equiv 0, \beta_{t}(r, 0)=\gamma(r)$ is written as $\beta(r, t)=[R(t) \gamma](r)$. If we multiply (4) by $\beta_{t}$, we get the energy identity

$$
\int\left(\beta_{t}^{2}+\beta_{r}^{2}+\frac{2}{r^{2}} \beta^{2}\right) d x=\int \gamma^{2} d x
$$

for all $t$. Thus $R(t)$ is bounded from $L^{2}\left(\mathbb{R}^{3}\right)$ into $\tilde{H}_{r}^{1}\left(\mathbb{R}^{3}\right)$ and $d R / d t$ from $L^{2}\left(\mathbb{R}^{3}\right)$ into $L^{2}\left(\mathbb{R}^{3}\right)$. Let $\alpha(r, t)$ be the given solution of $(1)$. Let $\alpha_{0}(r, t)$ be the free solution with the same Cauchy data at time $t=0$ as $\alpha(r, t)$ has. Then we have the integral form of (1)

$$
\alpha(t)=\alpha_{0}(t)+\int_{0}^{t} R(t-s)\left[\frac{3}{r} \alpha^{2}(s)-\alpha^{3}(s)\right] d s .
$$

Now we define

$$
\alpha_{+}(t)=\alpha_{0}(t)+\int_{0}^{\infty} R(t-s)\left[\frac{3}{r} \alpha^{2}(s)-\alpha^{3}(s)\right] d s .
$$

By Lemma 2 this integral converges in the norm of $\mathscr{H}$ and

$$
\left\|\alpha_{+}(t)-\alpha(t)\right\|_{*} \leqq \int_{t}^{\infty}\left\|\frac{3}{r} \alpha^{2}(s)-\alpha^{3}(s)\right\|_{L^{2}} d s
$$

tends to zero as $t \rightarrow \infty$. It is clear that, being a linear combination of free solutions, it is itself a free solution.

Q.E.D.

\section{The Representation and $L^{\infty}$-Estimates}

In Sect. 3 we will demonstrate the existence of the free-to-perturbed wave operators. For that purpose we need $L^{\infty}$-estimates on the solution $\alpha$ of $(1)$. Inverting the linear wave operator does not suffice for this purpose, because of the singularity in the term $\frac{2}{r^{2}} \alpha$. Therefore we will first find a fundamental solution for the operator $L$, given by (4).

Lemma 3. A classical solution $\alpha$ of the problem

$$
\begin{aligned}
L \alpha & =F(r, t) \quad \text { in }\{r>0, t>0\} \\
\alpha(r, 0) & =\alpha_{t}(r, 0)=0,
\end{aligned}
$$


is represented by

$$
\alpha(r, t)=\frac{\omega_{4}}{3 \omega_{5} r^{2}} \int_{0|r-t+\tau|}^{t} \int_{t+\tau}^{r+\tau} K(\rho, r, t-\tau) F(\rho, \tau) d \rho d \tau
$$

where the kernel $K$ is given by

$$
K(\rho, r, t)=\rho^{2}+r^{2}-t^{2},
$$

and where $\omega_{n}$ denotes the area of the unit sphere in $\mathbb{R}^{n}$. Thus, in the notation of the proof of Theorem 2,

$$
\alpha(t)=\int_{0}^{t} R(t-\tau) F(\tau) d \tau
$$

Proof. Introduce the change of variables

$$
\alpha=r v
$$

in (5). The Cauchy problem (5) then becomes

$$
v_{t t}-\frac{4}{r} v_{r}-v_{r r}=\frac{1}{r} F(r, t) \equiv \tilde{F}(r, t) .
$$

We recognize (9) as the nonhomogeneous five-dimensional radial wave equation, whose solution, as is well-known (cf. [2]), can be represented by

$$
v(r, t)=\frac{1}{3 !} \int_{0}^{t} d \tau \frac{\partial^{3}}{\partial t^{3}} \int_{0}^{t-\tau}\left[(t-\tau)^{2}-\rho^{2}\right] \rho Q(x, \rho, \tau) d \rho,
$$

where $Q(x, \rho, \tau)=\left(1 / \omega_{5}\right) \int_{|\omega|=1} \tilde{F}(x+\rho \omega, \tau) d \omega$.

When we carry out explicitly the differentiation indicated in (10), we get the result

$$
6 v(r, t)=\int_{0}^{t}\left[6(t-\tau) Q(x, t-\tau, \tau)+2(t-\tau)^{2} \frac{\partial Q}{\partial \rho}(x, t-\tau, \tau)\right] d \tau .
$$

Now we compute $Q$ explicitly, using the fact that $F$ (and hence $\tilde{F}$ ) is radial:

$$
Q(x, \rho, \tau)=\frac{\omega_{4}}{\omega_{5}} \int_{0}^{\pi} \tilde{F}\left(\left(|x|^{2}+\rho^{2}+2 \rho|x| \cos \phi\right)^{1 / 2}, \tau\right) \sin ^{3} \phi d \phi .
$$

Making the change of variable

$$
\lambda=\left(|x|^{2}+\rho^{2}+2 \rho|x| \cos \phi\right)^{1 / 2},
$$

we see that (12) is the same as

$$
Q(x, \rho, \tau)=\frac{\omega_{4}}{4 \omega_{5} \rho^{3}|x|^{3}} \int_{|\rho-| x||}^{\rho+|x|} \lambda\left[\lambda^{2}-(\rho-|x|)^{2}\right]\left[(\rho+|x|)^{2}-\lambda^{2}\right] \tilde{F}(\lambda, \tau) d \lambda .
$$

Next, we calculate $\partial Q / \partial \rho$ using $(12)^{\prime}$; the result is

$$
\frac{\partial Q}{\partial \rho}=\frac{-3}{\rho} Q+\frac{\omega_{4}}{\omega_{5} \rho^{2}|x|^{3}} \int_{|\rho-| x||}^{\rho+|x|} \lambda\left[\lambda^{2}-\rho^{2}+|x|^{2}\right] \tilde{F}(\lambda, \tau) d \lambda .
$$


Substitution of this in the formula (11) for $v$ gives

$$
v(r, t)=\frac{\omega_{4}}{3 \omega_{5}|x|^{3}} \int_{0}^{t} \int_{|| x \mid}^{|x|+t-\tau} \lambda K(\lambda,|x|, t-\tau) \tilde{F}(\lambda, \tau) d \lambda d \tau .
$$

Now $r \equiv|x|$. When we replace $v$ by $\alpha / r$ here, and recall that $\tilde{F}(r, t)=(1 / r) F(r, t)$, we obtain the statement of the lemma.

Corollary. The solution $\alpha_{0}$ of the linear problem

$$
L \alpha_{0}=0 ; \alpha_{0}(r, 0)=f(r), \partial_{t} \alpha_{0}(r, 0)=g(r)
$$

is represented by

$$
\alpha_{0}(r, t)=\frac{\omega_{4}}{3 \omega_{5} r^{2}} \int_{|r-t|}^{r+t} K(\rho, r, t) g(\rho) d \rho+\frac{\omega_{4}}{3 \omega_{5} r^{2}} \frac{\partial}{\partial t} \int_{|r-t|}^{r+t} K(\rho, r, t) f(\rho) d \rho .
$$

We now make the following standing hypotheses on the Cauchy data $f, g$ in (13):

$$
\begin{gathered}
\left(1+r^{2}\right) g(r) \in L^{2}\left(\mathbb{R}_{+}^{1}\right), r^{2} g \in L^{\infty}\left(\mathbb{R}_{+}^{1}\right) ; \\
r f(r) \in L^{\infty}\left(\mathbb{R}_{+}^{1}\right), r^{2} f^{\prime} \in L^{\infty}\left(\mathbb{R}_{+}^{1}\right), r f^{\prime} \in L^{1}\left(\mathbb{R}_{+}^{1}\right) \cap L^{2}\left(\mathbb{R}_{+}^{1}\right) .
\end{gathered}
$$

In making the $L^{\infty}$-estimates, we will need the following result, which is simply a calculus computation.

\section{Lemma 4.}

$$
\int_{t-r}^{t+r} \rho^{-2} K^{2}(\rho, r, t) d \rho=\frac{8}{3} r^{3}
$$

Define

$$
\begin{aligned}
& I_{g}=r^{-3 / 2} \int_{|r-t|}^{r+t} K(\rho, r, t) g(\rho) d \rho \\
& I_{f}=r^{-3 / 2} \frac{\partial}{\partial t} \int_{|r-t|}^{r+t} K(\rho, r, t) f(\rho) d \rho .
\end{aligned}
$$

Thus $\alpha_{0}=(c / \sqrt{r})\left(I_{g}+I_{f}\right)$.

Lemma 5. (Estimates on $I_{g}$ ). Assume $\left(H_{0}\right)$. Then
(a) $\left|I_{g}\right| \leqq c t^{-1 / 2}\left(\int_{|r-t|}^{r+t} \rho^{3} g^{2}(\rho) d \rho\right)^{1 / 2} \quad$ for all $r, t$
(b) $\left|I_{g}\right| \leqq c\left(\int_{|t-r|}^{t+r} \rho^{2} g^{2} d \rho\right)^{1 / 2} \quad$ for all $r, t$
(c) $\left|I_{g}\right| \leqq c t^{-1}\left(\int_{t-r}^{t+r} \rho^{4} g^{2} d \rho\right)^{1 / 2} \quad$ for $r \leqq \frac{t}{2}$
(d) $\left|I_{g}\right| \leqq c t^{-1} r^{1 / 2}\left\|\rho^{2} g\right\|_{L^{\infty}} \quad$ for $r \leqq \frac{t}{2}$ 
Proof. Call $a=|r-t|, b=r+t$. We may assume that $t>0$. We first establish (a) above in the case $r>t / 2$. For, splitting the kernel, we clearly have

$$
\begin{aligned}
\left|I_{g}\right| \leqq & r^{-3 / 2} \int_{a}^{b} \rho^{2}|g| d \rho+r^{-3 / 2}\left|r^{2}-t^{2}\right| \int_{a}^{b}|g| d \rho \\
\leqq & r^{-3 / 2}\left(\int_{a}^{b} \rho^{3} g^{2} d \rho\right)^{1 / 2}\left(\int_{a}^{b} \rho d \rho\right)^{1 / 2} \\
& +r^{-3 / 2}\left|r^{2}-t^{2}\right|\left(\int_{a}^{b} \rho^{3} g^{2} d \rho\right)^{1 / 2}\left(\int_{a}^{b} \rho^{-3} d \rho\right)^{1 / 2} \\
& \leqq c r^{-3 / 2}\left(\int_{a}^{b} \rho^{3} g^{2} d \rho\right)^{1 / 2}\left[r^{1 / 2} t^{1 / 2}+\left|r^{2}-t^{2}\right| \cdot \frac{r^{1 / 2} t^{1 / 2}}{\left|r^{2}-t^{2}\right|}\right] \\
\leqq & c t^{1 / 2} r^{-1}\left(\int_{a}^{b} \rho^{3} g^{2} d \rho\right)^{1 / 2} \leqq c t^{-1 / 2}\left(\int_{a}^{b} \rho^{3} g^{2} d \rho\right)^{1 / 2},
\end{aligned}
$$

since $r>t / 2$ by assumption. In order to prove (a) in the case $r<t / 2$, write

$$
\begin{aligned}
\left|I_{g}\right| & \leqq r^{-3 / 2} \int_{a}^{b}\left|\rho^{-3 / 2} K\right| \rho^{3 / 2}|g| d \rho \\
& \leqq r^{-3 / 2}\left(\int_{a}^{b} \rho^{-3} K^{2} d \rho\right)^{1 / 2}\left(\int_{a}^{b} \rho^{3} g^{2} d \rho\right)^{1 / 2}
\end{aligned}
$$

But for $r<t / 2, a=|t-r|=t-r \geqq t / 2$. Thus

$$
\begin{aligned}
\left|I_{g}\right| & \leqq c r^{-3 / 2} t^{-1 / 2}\left(\int_{a}^{b} \rho^{-2} K^{2} d \rho\right)^{1 / 2}\left(\int_{a}^{b} \rho^{3} g^{2} d \rho\right)^{1 / 2} \\
& \leqq c t^{-1 / 2}\left(\int_{a}^{b} \rho^{3} g^{2} d \rho\right)^{1 / 2},
\end{aligned}
$$

in view of Lemma 4 . This proves (a).

To establish (b) we write

$$
\begin{aligned}
\left|I_{g}\right| & =\left|r^{-3 / 2} \int_{a}^{b}\left(\frac{K}{\rho}\right) \rho g d \rho\right| \\
& \leqq r^{-3 / 2}\left(\int_{a}^{b} \rho^{-2} K^{2} d \rho\right)^{1 / 2}\left(\int_{a}^{b} \rho^{2} g^{2} d \rho\right)^{1 / 2} \\
& \leqq c\left(\int_{a}^{b} \rho^{2} g^{2} d \rho\right)^{1 / 2}
\end{aligned}
$$

by Lemma 4 . This proves (b).

Note that (a) implies (c) trivially, since $r \leqq t / 2$ is assumed in (c). Clearly (c) implies (d), which concludes the proof of Lemma 5 .

Lemma 5 contains the basic estimates which we need to solve the nonlinear 
problem. However, we digress to estimate the integral $I_{f}$, appearing in (16), to complete our study of the free equation.

Lemma 6. (Estimates on $I_{f}$ ).
(a) $\sup _{r \geqq 0}\left|I_{f}\right| \leqq c(1+t)^{-1 / 2}$
for all $t \geqq 0$
(b) $\sup _{r \leqq 1} r^{-1 / 2}\left|I_{f}\right| \leqq c(1+t)^{-1}$
for all $t \geqq 0$.

Proof. We compute $I_{f}$ explicitly, with the result

$$
\begin{aligned}
I_{f}= & 2 r^{-1 / 2}[(r+t) f(r+t)+|r-t| f(|r-t|)] \\
& -2 t r^{-3 / 2} \int_{|t-r|}^{t+r} f(\lambda) d \lambda .
\end{aligned}
$$

The last integral is, after integration by parts,

$$
\int_{|t-r|}^{t+r} f(\lambda) d \lambda=(t+r) f(t+r)-|t-r| f(|t-r|)-\int_{|t-r|}^{t+r} \lambda f^{\prime}(\lambda) d \lambda .
$$

Using this in (17), we obtain

$$
\begin{aligned}
I_{f}= & 2 r^{-3 / 2}\left[\left(r^{2}-t^{2}\right) f(t+r)+\left|r^{2}-t^{2}\right| f(|t-r|)\right] \\
& +2 t r^{-3 / 2} \int_{|t-r|}^{t+r} \lambda f^{\prime}(\lambda) d \lambda .
\end{aligned}
$$

In view of $\left(H_{0}\right)$, it is clear that $I_{f}=0\left(t^{-1 / 2}\right)$ for large $t$, provided $r>t / 2$. On the set $r<t / 2$, we have from (18)

$$
I_{f}=2 r^{-3 / 2}\left(t^{2}-r^{2}\right)[f(t-r)-f(t+r)]+2 t r^{-3 / 2} \int_{t-r}^{t+r} \lambda f^{\prime}(\lambda) d \lambda .
$$

The first term here is equal to

Hence $I_{f}$ can be written as

$$
-2\left(t^{2}-r^{2}\right) r^{-3 / 2} \int_{t-r}^{t+r} f^{\prime}(\lambda) d \lambda
$$

and so

$$
I_{f}=2 r^{-3 / 2} \int_{t-r}^{t+r}\left[t \lambda+r^{2}-t^{2}\right] f^{\prime}(\lambda) d \lambda
$$

$$
\begin{aligned}
\left|I_{f}\right| & \leqq 2 r^{1 / 2} \int_{t-r}^{t+r} \frac{1}{\lambda} \cdot \lambda\left|f^{\prime}\right| d \lambda+2 t r^{-3 / 2} \int_{t-r}^{t+r} \frac{|\lambda-t|}{\lambda^{2}} \cdot \lambda^{2}\left|f^{\prime}\right| d \lambda \\
& \leqq 2 r^{1 / 2}(t-r)^{-1}\left\|\lambda f^{\prime}\right\|_{L^{\prime}\left(\mathbb{R}^{1}\right)}+2 t r^{-3 / 2} \cdot \frac{r}{(t-r)^{2}} \int_{t-r}^{t+r} \lambda^{2}\left|f^{\prime}\right| d \lambda \\
& \leqq c r^{1 / 2} t^{-1}\left\|\lambda f^{\prime}\right\|_{L^{1}}+c t^{-1} r^{-1 / 2} \cdot\left\|\lambda^{2} f^{\prime}\right\|_{L^{\circ}} \cdot 2 r \\
& \leqq c r^{1 / 2} t^{-1} .
\end{aligned}
$$


This estimate completes the proof of (a) on the set $r<t / 2$, and establishes (b) simultaneously.

Corollary. Let $\alpha_{0}$ be a solution to the linear Cauchy problem (13) whose data satisfy $\left(H_{0}\right)$. Then we have the following estimates:
(a) $\sup _{r \geqq 0} r^{1 / 2}\left|\alpha_{0}(r, t)\right|=0\left(t^{-1 / 2}\right) \quad$ as $t \rightarrow \infty$.
(b) $\sup _{r \leqq 1}\left|\alpha_{0}(r, t)\right|=0\left(t^{-1}\right) \quad$ as $t \rightarrow \infty$.

Proof. The first estimate is a consequence of Lemma (5a) and Lemma (6a). The second conclusion follows immediately from Lemma (5d) and (6b).

We now turn to the $L^{\infty}$-estimates for the nonlinear problem. Let $\alpha_{-}(r, t)$ be a free solution $\left(L \alpha_{-}=0\right)$ with data given at time $t=-\infty$ which satisfy $\left(H_{0}\right)$. In Sect. 3 (to follow) we will convert (1) to integral form by inverting $L$, and will show that there exists a solution $\alpha(r, t)$ to the integral equation

$$
\alpha=\alpha_{-}+\frac{\omega_{4}}{3 \omega_{5} r^{2}} \int_{-\infty}^{t} \int_{a}^{b} K(\rho, r, t-\tau)\left(\frac{3 \alpha^{2}}{\rho}-\alpha^{3}\right) d \rho d \tau,
$$

where $a=|r-t+\tau|, b=r+t-\tau$, and $K$ is given by (7). We denote by $\mathscr{R} \alpha$ the operator mapping $\alpha$ into the right-hand side of (19). Let $T<0$. We will show that $\mathscr{R} \alpha$ is a contraction for sufficiently large $|T|$. The resulting solution $\alpha$ will be asymptotic to the given $\alpha_{-}$in the energy norm $\|\cdot\|_{*}$ as $t \rightarrow-\infty$. For our present purposes we define a norm by

$$
\begin{aligned}
\|\alpha\|_{0}= & \sup _{-\infty<t \leqq T}\left\{|t|^{1 / 2}\left\|r^{1 / 2} \alpha(r, t)\right\|_{L^{\infty}\left(\mathbb{R}^{3}\right)}\right. \\
& \left.+\frac{|t|}{\left|\ln ^{1 / 2}\right| t||}\left\|\frac{\alpha(r, t)}{r}\right\|_{L^{2}\left(\mathbb{R}^{3}\right)}\right\}
\end{aligned}
$$

Lemma 7. (A priori global $L^{\infty}$-estimate). If $\|\alpha\|_{0}<\infty$ on an interval $(-\infty, T]$, then the following estimate holds:

$$
\left\|r^{1 / 2}\left(\mathscr{R} \alpha-\alpha_{-}\right)\right\|_{\infty}<c\left(\|\alpha\|_{0}^{2}+\|\alpha\|_{0}^{3}\right)\left|\ln ^{1 / 2}\right| t \||t|^{-1}
$$

for all $t \in(-\infty, T)$.

Proof. From (19) we clearly have

$$
\left|r^{1 / 2}\left(\mathscr{R} \alpha-\alpha_{-}\right)\right| \leqq c \int_{-\infty}^{t}\left(\left|I^{\prime}\right|+\left|I^{\prime \prime}\right|\right) d \tau
$$


where

$$
\begin{aligned}
& I^{\prime}=r^{-3 / 2} \int_{a}^{b} K \cdot \frac{\alpha^{2}}{\rho} d \rho, \\
& I^{\prime \prime}=r^{-3 / 2} \int_{a}^{b} K \cdot \alpha^{3} d \rho .
\end{aligned}
$$

Note that these integrals are of precisely the same form as those treated in Lemma 5 (i.e. $I_{g}$ there). Hence, by Lemma (5a),

$$
\begin{aligned}
\left|I^{\prime}\right| & \leqq c(t-\tau)^{-1 / 2}\left(\int_{a}^{b} \rho^{3} \cdot \frac{\alpha^{4}}{\rho^{2}} d \rho\right)^{1 / 2} \\
& \leqq c(t-\tau)^{-1 / 2}\left\|r^{1 / 2} \alpha(\tau)\right\|_{\infty}\left(\int_{a}^{b} \alpha^{2} d \rho\right)^{1 / 2} \\
& \leqq c(t-\tau)^{-1 / 2}\left|\ln ^{1 / 2}\right| \tau|| \cdot|\tau|^{-3 / 2}\|\alpha\|_{0}^{2}
\end{aligned}
$$

It follows that

$$
\begin{aligned}
\int_{-\infty}^{t}\left|I^{\prime}\right| d \tau & \leqq c\|\alpha\|_{0}^{2} \int_{-\infty}^{t}(t-\tau)^{-1 / 2}\left|\ln ^{1 / 2}\right| \tau|| \cdot|\tau|^{-3 / 2} d \tau \\
& \leqq c\|\alpha\|_{0}^{2}|t|^{-1}\left|\ln ^{1 / 2}\right| t||,
\end{aligned}
$$

which is the required estimate for this term. To treat $I^{\prime \prime}$, apply Lemma (5b):

$$
\begin{aligned}
\left|I^{\prime \prime}\right| \leqq c\left(\int_{a}^{b} \rho^{2} \alpha^{6} d \rho\right)^{1 / 2} & \leqq c\left\|r^{1 / 2} \alpha(\tau)\right\|_{\infty}^{2}\left(\int_{a}^{b} \alpha^{2} d \rho\right)^{1 / 2} \\
& \leqq c\|\alpha\|_{0}^{3} \tau^{-2}\left|\ln ^{1 / 2}\right| \tau \| .
\end{aligned}
$$

Thus

$$
\begin{aligned}
\int_{-\infty}^{t}\left|I^{\prime \prime}\right| d \tau & \leqq c\|\alpha\|_{0}^{3} \int_{-\infty}^{t} \frac{\left|\ln ^{1 / 2}\right| \tau|| d \tau}{\tau^{2}} \\
& \leqq c\|\alpha\|_{0}^{3} \frac{\left|\ln ^{1 / 2}\right| t||}{|t|}
\end{aligned}
$$

as desired.

Lemma 8. (A priori local $L^{\infty}$-estimates). Let $\|\alpha\|_{0}<\infty$ on an interval $(-\infty, T]$. Then the following estimates hold:

(a) $r^{1 / 2}\left|\mathscr{R} \alpha-\alpha_{-}\right| \leqq c r^{1 / 4}\left(\|\alpha\|_{0}^{2}+\|\alpha\|_{0}^{3}\right)\left|\ln ^{1 / 4}\right| t|||t|^{-1} \quad$ for $r \leqq 1, t \in(-\infty, T]$.

(b) For $r \leqq 1, t \in(-\infty, T],\left|\mathscr{R} \alpha-\alpha_{-}\right| \leqq c\left(\|\alpha\|_{0}^{2}+\|\alpha\|_{0}^{3}\right)|t|^{-1} \times$

$$
\left[1+\left|\ln \left(\frac{2 r}{2 r+|t|}\right)\right|\right] \text {. }
$$


Proof. We split up the integral operator (19) as

$$
\begin{aligned}
r^{1 / 2}\left(\mathscr{R} \alpha-\alpha_{-}\right) & =\frac{c}{r^{3 / 2}}\left[\int_{-\infty}^{t-2 r}+\int_{t-2 r}^{t}\right]_{a}^{b} K\left(\frac{3 \alpha^{2}}{\rho}-\alpha^{3}\right) d \rho d \tau \\
& \equiv E_{1}+E_{2} .
\end{aligned}
$$

Consider $E_{2}$. By applying Lemma (5a) as above, we find

$$
\begin{aligned}
\left|E_{2}\right| & \leqq c \int_{t-2 r}^{t}(t-\tau)^{-1 / 2}\left[\left(\int_{a}^{b} \rho^{3} \cdot \frac{\alpha^{4}}{\rho^{2}} d \rho\right)^{1 / 2}+\left(\int_{a}^{b} \rho^{3} \alpha^{6} d \rho\right)^{1 / 2}\right] d \tau \\
& \leqq c \int_{t-2 r}^{t}(t-\tau)^{-1 / 2}\left[\left|\ln ^{1 / 2}\right| \tau||\|\alpha\|_{0}^{2}|\tau|^{-3 / 2}+\left\|r^{1 / 2} \alpha(\tau)\right\|_{\infty}^{3}\left(\int_{a}^{b} d \rho\right)^{1 / 2}\right] d \tau .
\end{aligned}
$$

Here we have used on the first term the estimate for $I^{\prime}$ in Lemma 7. Therefore

$$
\begin{aligned}
\left|E_{2}\right| \leqq & c\|\alpha\|_{0}^{2}\left|\ln ^{1 / 2}\right| t|||t|^{-3 / 2} r^{1 / 2} \\
& +c\|\alpha\|_{0}^{3} r^{1 / 2} \int_{t-2 r}^{t}|\tau|^{-3 / 2}(t-\tau)^{-1 / 2} d \tau,
\end{aligned}
$$

since $r \leqq 1$. Hence we find

$$
\left|E_{2}\right| \leqq c\left(\|\alpha\|_{0}^{2}+\|\alpha\|_{0}^{3}\right) r^{1 / 2}\left|\ln ^{1 / 2}\right| t|||t|^{-3 / 2}
$$

for $r \leqq 1$.

To handle $E_{1}$, we apply Lemma $(5 \mathrm{c})$ :

$$
\left|E_{1}\right| \leqq c \int_{-\infty}^{t-2 r}(t-\tau)^{-1}\left[\left(\int_{a}^{b} \rho^{4} \cdot \frac{\alpha^{4}}{\rho^{2}} d \rho\right)^{1 / 2}+\left(\int_{a}^{b} \rho^{4} \cdot \alpha^{6} d \rho\right)^{1 / 2}\right] d \tau
$$

The second term here can be bounded as

$$
\begin{aligned}
\left(\int_{a}^{b} \rho^{4} \alpha^{6} d \rho\right)^{1 / 2} & \leqq\left\|r^{1 / 2} \alpha(\tau)\right\|_{\infty}^{3}\left(\int_{a}^{b} \rho d \rho\right)^{1 / 2} \\
& \leqq c\|\alpha\|_{0}^{3}|\tau|^{-3 / 2} r^{1 / 2}(t-\tau)^{1 / 2}
\end{aligned}
$$

The first term we bound as

$$
\begin{aligned}
\left(\int_{a}^{b} \rho^{2} \alpha^{4} d \rho\right)^{1 / 2} & \leqq\left\|r^{1 / 2} \alpha(\tau)\right\|_{\infty}^{3 / 2}\left(\int_{a}^{b} \alpha^{2} \mathrm{~d} \rho\right)^{1 / 4}\left(\int_{a}^{b} \rho d \rho\right)^{1 / 4} \\
& \leqq c\|\alpha\|_{0}^{2}\left|\ln ^{1 / 4}\right| \tau|||\tau|^{-5 / 4} r^{1 / 4}(t-\tau)^{1 / 4}
\end{aligned}
$$

Using (23), (24) in the estimate (22) for $E_{1}$, we find

$$
\begin{aligned}
\left|E_{1}\right| \leqq & c \int_{-\infty}^{t-2 r}(t-\tau)^{-1}\left[\|\alpha\|_{0}^{2}\left|\ln ^{1 / 4}\right| \tau|||\tau|^{-5 / 4} r^{1 / 4}(t-\tau)^{1 / 4}\right. \\
& \left.+\|\alpha\|_{0}^{3}|\tau|^{-3 / 2} r^{1 / 2}(t-\tau)^{1 / 2}\right] d \tau \\
\leqq & c r^{1 / 4}\left(\|\alpha\|_{0}^{2}+\|\alpha\|_{0}^{3}\right)\left|\ln ^{1 / 4}\right| t|||t|^{-1}
\end{aligned}
$$


since $r \leqq 1$. This and the estimate (21) for $E_{2}$ establish part (a) of Lemma 8 .

It remains to prove part (b). Notice that the estimate (21) for $E_{2}$ is stronger than that claimed in part (b) of Lemma 8 . Therefore we need only estimate $E_{1}$. Since the second integral in (22) is dominated by $c\|\alpha\|_{0}^{3} r^{1 / 2}|t|^{-1}$, as follows from (23), we can write

$$
\left|E_{1}\right| \leqq c\left(\|\alpha\|_{0}^{2}+\|\alpha\|_{0}^{3}\right) r^{1 / 2}|t|^{-1}+c \int_{-\infty}^{t-2 r}(t-\tau)^{-1}\left(\int_{a}^{b} \rho^{2} \alpha^{4} d \rho\right)^{1 / 2} d \tau
$$

The last integral is less than

$$
\begin{aligned}
\int_{-\infty}^{t-2 r}(t-\tau)^{-1}\left\|r^{1 / 2} \alpha(\tau)\right\|_{\infty}^{2}\left(\int_{a}^{b} d \rho\right)^{1 / 2} d \tau & \leqq c\|\alpha\|_{0}^{2} r^{1 / 2} \int_{-\infty}^{t-2 r} \frac{d \tau}{|\tau|(t-\tau)} \\
& \leqq c\|\alpha\|_{0}^{2} r^{1 / 2}|t|^{-1}\left|\ln \left(\frac{2 r}{2 r+|t|}\right)\right|
\end{aligned}
$$

and this establishes part (b).

\section{The Free-to-Perturbed Wave Operators}

Let $\alpha_{-}(r, t)$ be a free solution $\left(L \alpha_{-}=0\right)$ with data given at time $t=-\infty$ which satisfy $\left(H_{0}\right)$. Let $-\infty<T<0$, and let

$$
\begin{gathered}
a=|r-t+\tau|, b=r+t-\tau, \\
K(\rho, r, t)=\rho^{2}+r^{2}-t^{2} .
\end{gathered}
$$

We wish to show that there exists a unique solution $\alpha(r, t)$ of the nonlinear equation

$$
\alpha_{t t}-\frac{2}{r} \alpha_{r}-\alpha_{r r}+\frac{2}{r^{2}} \alpha-\frac{3 \alpha^{2}}{r}+\alpha^{3}=0
$$

such that

$$
\left\|\alpha(t)-\alpha_{-}(t)\right\|_{*} \rightarrow 0 \text { as } t \rightarrow-\infty
$$

Here the energy norm $\|\cdot\|_{*}$ is given by (2).

The existence of such an $\alpha$ will be achieved by first showing that the integral equation,

$$
\alpha(t)=\alpha_{-}(t)+\frac{\omega_{4}}{3 \omega_{5} r^{2}} \int_{-\infty}^{t} \int_{a}^{b} K(\rho, r, t-\tau)\left(\frac{3 \alpha^{2}}{\rho}-\alpha^{3}\right) d \rho d \tau \equiv \mathscr{R} \alpha,
$$

has a unique solution on the interval $-\infty<t \leqq T$, provided $|T|$ is sufficiently large. This in turn follows from our showing below that the operator $\mathscr{R}$ on the right-hand side of (19) is a contraction for large enough $|T|$. The solution so obtained will be shown to exist for all times $t \in \mathbb{R}$, as follows from positivity of the energy.

Define a norm on functions $\alpha(r, t)(0 \leqq r<\infty,-\infty<t \leqq T)$ by

$$
\|\alpha\|=\sup _{-\infty<t \leqq T}\left\{\sup _{r}|\sqrt{r|t|}| \alpha\left|+\sup _{r \leqq 1}\right| \frac{t}{\ln t} \frac{\alpha}{\ln \left(\frac{2 r}{2 r+|t|}\right)} \mid\right.
$$




$$
\left.+\frac{|t|}{\sqrt{\ln |t|}}\left\|\frac{\alpha}{r}\right\|_{2}+\|\alpha\|_{*}+\|\alpha\|_{2}+|t|^{2 / 3}\left\|r^{-1 / 3} \alpha\right\|_{3}\right\} .
$$

All spatial norms here are to be taken over $\mathbb{R}^{3}$. It follows from the corollary to Lemma 6 and from estimates to be made below that $\||\alpha|\|<\infty$ for a free solution with data satisfying $\left(H_{0}\right)$.

Theorem 3. Let $\alpha_{-}(r, t)$ be a solution of the free equation $L \alpha_{-}=0$, whose data satisfy $\left(H_{0}\right)$. Then there exists a unique solution $\alpha$ of the perturbed equation

$$
L \alpha-\frac{3 \alpha^{2}}{r}+\alpha^{3}=0
$$

such that $\left\|\alpha(t)-\alpha_{-}(t)\right\|_{*} \rightarrow 0$ as $t \rightarrow-\infty$. Moreover, this perturbed solution enjoys the following properties:

(i) $\alpha \in C^{0}\left(\mathbb{R}, \tilde{H}_{r}^{1}\right) ; \alpha_{t} \in C^{0}\left(\mathbb{R}, L_{r}^{2}\right)$,

(ii) the total energy is conserved:

$\int_{\mathbb{R}^{3}}\left[\frac{1}{2} \alpha_{t}^{2}+\frac{1}{2}|\nabla \alpha|^{2}+\frac{1}{4} \alpha^{2}\left(\alpha-\frac{2}{r}\right)^{2}\right] d x=\frac{1}{2}\left\|\alpha_{-}\right\|_{*}^{2}=$ constant,

(iii) ||$\alpha|| \mid<\infty$.

Proof. Consider the integral equation (19) on the interval $(-\infty, T)$. We will prove that there exists a solution $\alpha$ of (19) for $|T|$ large, and such a solution is certainly a weak solution of (1). Notice that $\|\alpha\|_{0} \leqq\|\alpha\| \|$ and that the $L^{\infty}$-estimates for $\mathscr{R} \alpha-\alpha$ in the first two terms of $\||\cdot|\|($ (cf. (25)) have already been given in Lemmas 7 and 8b).

Lemma 9. Let $\|\alpha \mid\|<\infty$ on an interval $(-\infty, T]$. Then there exists a constant $c$ such that

$$
\sup _{-\infty<t \leqq T}\left\|\mathscr{R} \alpha(t)-\alpha_{-}(t)\right\|_{*} \leqq c|T|^{-1 / 2}\left|\ln ^{1 / 2}\right| T \|\left(\|\| \alpha\left\|\left.\right|^{2}+\right\| \alpha \mid \|^{3}\right) .
$$

Proof. First we note that, given a solution $u$ of $L u=f$, where $f \in L_{\text {loc }}^{1}\left(\mathbb{R}, L_{r}^{2}\right)$, with zero Cauchy data, we have

$$
\|u(t)\|_{*} \leqq c \int_{0}^{t}\|f(\tau)\|_{2} d \tau
$$

as follows from the proof of Theorem 2.

Hence, by applying (27) to (19), we get

$$
\left\|\mathscr{R} \alpha(t)-\alpha_{-}(t)\right\|_{*} \leqq c \int_{-\infty}^{t}\left\|\left(\frac{3 \alpha^{2}}{r}-\alpha^{3}\right)\right\|_{2} d \tau
$$

Now $\left\|\alpha^{3}\right\|_{2} \leqq\left\|r^{1 / 2} \alpha\right\|_{\infty}^{2}\|\alpha / r\|_{2} \leqq c\left|\left\|\left.\alpha\left|\|^{3}\right| \tau\right|^{-2}\left|\ln ^{1 / 2}\right| \tau||\right.\right.$, and

$$
\left\|\frac{\alpha^{2}}{r}\right\|_{L^{2}(r>1)} \leqq\left\|r^{1 / 2} \alpha\right\|_{\infty}\left\|\frac{\alpha}{r}\right\|_{2^{r} \geqq 1} \sup ^{-1 / 2} \leqq c\||\alpha|\|^{2}\left|\ln ^{1 / 2}\right| \tau|||\tau|^{-3 / 2} \text {. }
$$


The estimate for this term near the origin is made by using Lemma 8 b):

$$
\begin{aligned}
& \left\|\frac{\alpha^{2}}{r}\right\|_{L^{2}(r \leqq 1)}=c\left(\int_{0}^{1} \alpha^{4} d r\right)^{1 / 2} \\
& =c\left(\int_{0}^{1} \frac{\tau^{4} \alpha^{4}}{\ln ^{4}|\tau| \ln ^{4}\left(\frac{2 r}{2 r+|\tau|}\right)} \cdot \frac{\ln ^{4}|\tau| \cdot \ln ^{4}\left(\frac{2 r}{2 r+|\tau|}\right) d r}{\tau^{4}}\right)^{1 / 2} \\
& \leqq c\|\alpha\|^{2} \frac{\ln ^{2}|\tau|}{\tau^{2}}\left(\int_{0}^{1} \ln ^{4}\left(\frac{2 r}{2 r+|\tau|}\right) d r\right)^{1 / 2} \leqq\|\alpha\|^{2} \ln ^{4}|\tau| \cdot \tau^{-2}
\end{aligned}
$$

Using these estimates in (28) and integrating in $\tau$, we obtain (26).

To complete the estimation of $\left\|\mathscr{R} \alpha-\alpha_{-}\right\| \|$, we need the following $L^{p}$-estimates:

Lemma 10. Let $\beta$ be a solution of $L \beta=f$ in $\{t>0\}$ having zero Cauchy data. Then

(i) $\left\|r^{-1 / 3} \beta\right\|_{L^{3}\left(\mathbb{R}^{3}\right)} \leqq c \int_{0}^{t}(t-\tau)^{-2 / 3}\left\|r^{1 / 3} f(\tau)\right\|_{L^{3 / 2}\left(\mathbb{R}^{3}\right)} d \tau ;$

(ii) $\|\beta\|_{L^{2}\left(\mathbb{R}^{3}\right)} \leqq c \int_{0}^{t}\left\|r^{2 / 5} f(\tau)\right\|_{L^{10 / 7}\left(\mathbb{R}^{3}\right)} d \tau$.

Proof. As in Sect. 2, Eq. (8), introduce the change of variables $\beta=r v$ into the equation $L \beta=f$. The equation for $v$ becomes

$$
v_{t t}-\frac{4}{r} v_{r}-v_{r r}=r^{-1} f
$$

and the operator on the left-hand side here is again the five-dimensional wave operator. Then as a special case of the estimates proved in [7], we conclude

$$
\|v(t)\|_{\left.L^{3(\mathbb{R})}\right)} \leqq c \int_{0}^{t}(t-\tau)^{-2 / 3}\left\|r^{-1} f(\tau)\right\|_{L^{3 / 2(\mathbb{R} 5)}} d \tau
$$

and

$$
\|v(t)\|_{L^{2}\left(\mathbb{R}^{5}\right)} \leqq c \int_{0}^{t}\left\|r^{-1} f(\tau)\right\|_{L^{10 / 7}\left(\mathbb{R}^{5}\right)} d \tau .
$$

Since $\beta \equiv r v$, we then have

$$
\|v(t)\|_{L^{3}\left(\mathbb{R}^{5}\right)}=\left\|r^{-1 / 3} \beta(t)\right\|_{L^{3}\left(\mathbb{R}^{3}\right)},
$$

and

$$
\|v(t)\|_{L^{2}\left(\mathbb{R}^{5}\right)}=\|\beta(t)\|_{L^{2}\left(\mathbb{R}^{3}\right)},
$$

and these identifications complete the proof.

Lemma 11. Let $\|\alpha\| \|<$ on an interval $(-\infty, T]$. Then there exists a constant $c$ such 
that

$$
\left\|r^{-1 / 3}\left(\mathscr{R} \alpha-\alpha_{-}\right)(t)\right\|_{3} \leqq c\left(\left.\left|\left\|\alpha||^{2}+\left.|\| \alpha|\right|^{3}\right)\right| t\right|^{-1} \text { for }-\infty<t \leqq T\right. \text {. }
$$

Proof. Applying i) of Lemma 10 to (19), we find

$$
\left\|r^{-1 / 3}\left(\mathscr{R} \alpha-\alpha_{-}\right)(t)\right\|_{3} \leqq c \int_{-\infty}^{t}(t-\tau)^{-2 / 3}\left\|\left(\frac{-3 \alpha^{2}}{r}+\alpha^{3}\right) r^{1 / 3}(\tau)\right\|_{3 / 2} d \tau .
$$

Now $\left\|r^{1 / 3} \alpha^{2} / r\right\|_{3 / 2}=\left\|r^{-1 / 3} \alpha\right\|_{3}^{2} \leqq c|\tau|^{-4 / 3} \mid\|\alpha\|$, and

$$
\begin{aligned}
\left\|r^{1 / 3} \alpha^{3}\right\|_{3 / 2} & \leqq\left\|r^{1 / 2} \alpha\right\|_{\infty}^{4 / 3}\|\alpha\|_{2}^{2 / 3}\left\|r^{-1 / 3} \alpha\right\|_{3} \\
& \leqq\left. c\left|\|\alpha\|^{3}\right| \tau\right|^{-4 / 3}
\end{aligned}
$$

Putting these estimates together, we obtain

$$
\left\|r^{-1 / 3}\left(\mathscr{R} \alpha-\alpha_{-}\right)(t)\right\|_{3} \leqq c\left(|\|\alpha\||^{2}+\||\alpha|\|^{3}\right) \int_{-\infty}^{t}(t-\tau)^{-2 / 3}|\tau|^{-4 / 3} d \tau,
$$

and this proves the Lemma.

Lemma 12. Let $\|\alpha\| \|<$ on an interval $(-\infty, T]$. Then there exists a constant $c$ such that

$$
\left\|\left(\mathscr{R} \alpha-\alpha_{-}\right)(t)\right\|_{2} \leqq c\left(\left\|\left.|\alpha|\right|^{2}+\right\||| \alpha \|^{3}\right)|t|^{-1 / 5} \quad \text { for }-\infty<t \leqq T .
$$

Proof. Applying ii) of Lemma 10 to (19), we find

$$
\left\|\left(\mathscr{R} \alpha-\alpha_{-}\right)(t)\right\|_{2} \leqq c \int_{-\infty}^{t}\left\|r^{2 / 5}\left(\frac{-3 \alpha^{2}}{r}+\alpha^{3}\right)(\tau)\right\|_{10 / 7} d \tau
$$

Now

$$
\left\|r^{2 / 5} \cdot \frac{\alpha^{2}}{r}\right\|_{10 / 7} \leqq\|\alpha\|_{2}^{1 / 5}\left\|r^{-1 / 3} \alpha\right\|_{3}^{9 / 5} \leqq c\left|\left\|\left.\alpha\left|\|^{2}\right| \tau\right|^{-6 / 5}\right.\right.
$$

and

$$
\begin{aligned}
\left\|r^{2 / 5} \alpha^{3}\right\|_{10 / 7} & \leqq\left\|r^{1 / 2} \alpha\right\|_{\infty}^{4 / 3}\|\alpha\|_{2}^{13 / 15}\left\|r^{-1 / 3} \alpha\right\|_{3}^{4 / 5} \\
& \leqq c\left|\left\|\left.\alpha\left|\|^{3}\right| \tau\right|^{-6 / 5}\right.\right.
\end{aligned}
$$

Putting these estimates into (31), we obtain the result of the lemma.

There is only one more term in the norm $\| \mathscr{R} \alpha-\alpha_{-}|| \mid$to be estimated. This is the content of

Lemma 13. Let $\||\alpha|\|<\infty$ on an interval $(-\infty, T]$. Then there exists a constant $c$ such that

$$
\left.\left\|r^{-1}\left(\mathscr{R} \alpha-\alpha_{-}\right)(t)\right\|_{L^{2}\left(\mathbb{R}^{3}\right)} \leqq c|\|\alpha\||^{2}+\||| \alpha||^{3}\right)\left|\ln ^{1 / 4}\right| t|||t|^{-1}
$$

for $t \in(-\infty, T]$.

Proof. This result follows from already derived bounds. First, let

$$
J_{1}=\int_{r \geqq 1} r^{-2}\left|\mathscr{R} \alpha-\alpha_{-}\right|^{2} d x .
$$


Then

$$
\begin{aligned}
J_{1} & \leqq\left\|r^{-1 / 3}\left(\mathscr{R} \alpha-\alpha_{-}\right)(t)\right\|_{3}^{2}\left(\int_{r \geqq 1} r^{-4} d x\right)^{1 / 3} \\
& \leqq c\left(\|||\|^{2}+\|\mid \alpha\|^{3}\right)^{2} t^{-2},
\end{aligned}
$$

where we have used (29) from Lemma 11. Hence

$$
\left\|r^{-1}\left(\mathscr{R} \alpha-\alpha_{-}\right)(t)\right\|_{L^{2}(r \geqq 1)} \leqq c\left(\left\|\left.||\left|\left\|^{2}+\right\|\right||\alpha|\right|^{3}\right)|t|^{-1},\right.
$$

and this is stronger than the desired estimate (32). For the estimate on the complement $\{r<1\}$, we have

$$
\left|J_{2}\right| \equiv \int_{r<1} \frac{\left(\mathscr{R} \alpha-\alpha_{-}\right)^{2}}{r^{2}} d^{3} x=\int_{r<1} \frac{r\left|\mathscr{R} \alpha-\alpha_{-}\right|^{2}}{r^{3}} d^{3} x .
$$

Now bound $r\left|\mathscr{R} \alpha-\alpha_{-}\right|^{2}$ using a) of Lemma 8 :

$$
\begin{aligned}
\left|J_{2}\right| & \leqq c \int_{r<1} r^{-3} \cdot r^{1 / 2}\left(\||\alpha|\|^{2}+\|\left.|\alpha|\right|^{3}\right)^{2}\left|\ln ^{1 / 2}\right| t|| t^{-2} d^{3} x \\
& \leqq c\left(\||\alpha|\|^{2}+\||| \alpha \mid\|^{3}\right)^{2}\left|\ln ^{1 / 2}\right| t|| t^{-2} \int_{0}^{1} r^{-1 / 2} d r .
\end{aligned}
$$

Therefore

$$
\left\|r^{-1}\left(\mathscr{R} \alpha-\alpha_{-}\right)(t)\right\|_{L^{2}(r \leqq 1)} \leqq c\left(\left\|\alpha\left|\left\|^{2}+\right\|\right| \alpha \mid\right\|^{3}\right) \ln ^{1 / 4}|t| \|\left. t\right|^{-1} .
$$

This establishes Lemma 13.

If we now examine the results of Lemmas 7-13, we conclude the following:

Lemma 14. Let $\||\alpha|\|<\infty$ on an interval $(-\infty, T]$. Then there exists a constant $c$ such that

$$
|| \mathscr{R} \alpha-\alpha_{-}|||\leqq c| \ln ^{-1 / 4}|T| \mid\left(\left|\left\|\alpha||^{2}+\left.|\| \alpha|\right|^{3}\right)\right.\right.
$$

It is now clear from the methods of Lemmas 9-13 and the corollary to Lemma 6 that \|\|$\alpha_{-} \| \mid<\infty$ for a free solution $\alpha_{-}$with data satisfying $\left(H_{0}\right)$. Inequality (34) shows that the operator $\alpha \rightarrow \mathscr{R} \alpha$ given by (19) is a contraction for $|T|$ sufficiently large. Thus there exists a time $T$ and a unique solution $\alpha=\alpha(r, t)$ of the integral equation (19) for $-\infty<t<T<0$. By construction and the definition (25) of the norm $\|\cdot \mid\|$ we see that

$$
\begin{aligned}
& \alpha \in C\left((-\infty, T) ; \tilde{H}_{r}^{1}\right), \text { and } \\
& \alpha_{t} \in C\left((-\infty, T) ; L_{r}^{2}\right) .
\end{aligned}
$$

This solution $\alpha$ can be continued for all times $-\infty<t<\infty$. This follows exactly as in [6] (from the positive definite nature of the energy density e). By (28), we have, since $\mathscr{R} \alpha=\alpha$,

$$
\left\|\left(\alpha-\alpha_{-}\right)(t)\right\|_{*} \leqq c \int_{-\infty}^{t}\left\|\left(\frac{3 \alpha^{2}}{r}-\alpha^{3}\right)(\tau)\right\|_{2} d \tau,
$$

and the result of Lemma 9 shows that this integral tends to zero as $t \rightarrow-\infty$. 


\section{References}

1. Choquet-Bruhat, Y., Christodoulou, D.: Existence of global solutions of the Yang-Mills, Higgs and spinor field equations in $3+1$ dimensions, (to appear)

2. Courant, R., Hilbert, D.: Methods of mathematical physics, Volume II, New York: Interscience 1962

3. Eardley, D., Moncrief, V.: The global existence of Yang-Mills-Higgs fields in 4-dimensional Minkowski space. Commun. Math. Phys. 83, 171-212 (1982)

4. Ginibre, J., Velo, G.: The Cauchy problem for coupled Yang-Mills and scalar fields in the temporal gauge. Commun. Math. Phys. 82, 1-28 (1981),... in the Lorentz gauge (to appear)

5. Glassey, R., Strauss, W.: Decay of classical Yang-Mills fields, Commun. Math. Phys. 65, 1-13 (1979)

6. Glassey, R., Strauss, W.: Some global solutions of the Yang-Mills equations in Minkowski space. Commun. Math. Phys. 81, 171-187 (1981)

7. Marshall, B., Strauss, W., Wainger, S.: $L^{p}-L^{q}$ estimates for the Klein-Gordon equation, J. Math. Pures Appl. 59, 417-440 (1980)

8. Segal, I.: The Cauchy problem for the Yang-Mills equations. J. Funct. Anal. 33, 175-194 (1979)

Communicated by A. Jaffe

Received November 16, 1982, in revised form January 28, 1983. 